www.nature.com/jes

\title{
EDITORIAL
}

\section{Bringing exposure science into the 21st century}

Journal of Exposure Science and Environmental Epidemiology (2009) 19, 535; doi:10.1038/jes.2009.37

Exposure science continues to be a growing discipline whose face is ever evolving. In the past few decades, this field has become a major component of most environmentally related studies or has contributed in some manner to risk assessment and risk management. Major advances in technologies have made the generation of data a much easier task than it was earlier. Also, with growing concerns over ethical issues in science and limited financial resources, we have had to be innovative in our approaches to furthering our understanding of exposure and toxicity, and their application in the risk assessment process. The new face of exposure science will include a multitude of techniques, such as high throughput toxicity testing, that are both cost effective and provide a wider array of information in a shorter period of time. As biomonitoring studies continue to grow in both scale and importance, we need to focus resources and efforts on better understanding of the data generated from these studies. In the twenty-first century, we have to maximize the use of our limited research funds to not only provide the maximum information for evaluating exposure or toxicity but also provide the appropriate framework for understanding these data. Only when we combine these two will we be able to fully use the power of exposure science.

We are fortunate to have an issue of the Journal of Exposure Science and Environmental Epidemiology that is focused on furthering exposure science and maximizing the utility of the information we glean from each study. This special issue was generously funded through an unrestricted grant by the International Council of Chemical Association's Long-Range Research Initiative (ICCA-LRI) in collaboration with the International Society of Exposure Science. Hopefully, this compilation of articles will offer our readership some insight into both successes and challenges we face within the field and the environmental community at large.

DANA B. BARR Editor-in-Chief 